\title{
Desempenho e atitudes em relação à matemática de alunos do 6 ano do ensino fundamental
}

Cicero Luciano Alves Costa

Cicera Vania Barros Costa

\begin{abstract}
Resumo
O objetivo do trabalho foi investigar associações entre as atitudes em relação à Matemática e o desempenho na disciplina de alunos do 6ㅇ ano do Ensino Fundamental. Participaram 37 escolares com idades entre 10 e 14 anos. Utilizouse a Escala de Atitudes em Relação à Matemática (EARM) para avaliar as atitudes dos alunos em relação à disciplina. $O$ desempenho na disciplina de Matemática foi investigado a partir da média das notas dos três primeiros bimestres. As meninas apresentaram médias superiores aos meninos tanto na EARM quanto no desempenho escolar. $O$ teste de correlação de Spearman apontou correlação positiva e moderada. Quando tal associação foi analisada separadamente, observou-se que nos meninos a correlação foi fraca (ró=0,343), enquanto que nas meninas encontrou-se forte $(r o ́=0,758)$. Os achados evidenciam uma relação positiva entre as atitudes em relação à Matemática e o desempenho escolar na disciplina.
\end{abstract}

Palavras-chave: Atitudes em relação à Matemática, Ensino Fundamental, Desempenho Escolar.

\begin{abstract}
The objective of this study was to evaluate possible associations between attitudes toward mathematics and performance in the discipline of students in the 6th grade of elementary school. Participants were 37 students aged between 10 and 14 years. We used the Scale of Attitudes Toward Mathematics (MRSA) to assess students' attitudes towards discipline. The school performance in Mathematics was investigated from the average grade of the first three marking periods. Girls had higher means for boys in both MRSA and performance. The correlation test of Spearman was positive and moderate. When this association was examined separately, showed that in boys the correlation was weak ( $r$ ho = 0.343), while the girls met strong ( $r h o=0.758$ ). The findings go against the literature, showing a positive relationship between attitudes toward mathematics and academic performance in the discipline.
\end{abstract}

Keywords: Attitudes Toward Mathematics, Elementary School, School Performance. 


\section{Introdução}

Há uma grande preocupação nas escolas de Ensino Básico em relação ao aprendizado dos alunos na disciplina de Matemática (FIORENTINI e MIORIM, 1990; SANCHEZ, 2004). Segundo Loos et al. (2001), a Matemática é a disciplina campeã no que diz respeito aos índices de reprovação escolar. Ainda mais, entre os alunos, do Ensino Fundamental ao Médio, essa é a disciplina que provoca maior aversão.

Como mostram os psicólogos contemporâneos, o desenvolvimento da aprendizagem não depende somente de fatores inatos (de ordem genética), nem tão pouco de aspectos ambientais (fenótipo), mas sim da associação dos fatores biopsicossociais (KREBS, 2004). Ainda mais, para que ocorra um aprendizado efetivo do aluno é preciso levar em conta, além do aspecto cognitivo, os fatores afetivos e emocionais.

Desta forma, a participação e acompanhamento dos pais e da família tornam-se relevantes para o processo educacional no que concerne à motivação e incentivo dado às crianças (PAULA, 2008). Conforme Brito (2002), os fatores afetivos e emocionais irão influenciar o entendimento dos conteúdos aprendidos e depois recordados qualitativamente e quantitativamente. À escola, cabe o papel de oportunizar situações capazes de promover o desenvolvimento do aluno. Assim, é papel do professor tornar o momento de aprendizado agradável, capaz de incentivar e motivar o aluno. Ainda mais, os professores que conhecem as atitudes dos seus alunos têm mais chances de melhorar o desempenho dos mesmos, pois conseguem refletir sobre as experiências que os alunos já tiveram dentro da disciplina (PAULA, 2008).

Baseando-se em estudos desenvolvidos na área da Educação Matemática e a partir da observação informal que a Matemática para alguns é vista (como uma disciplina que causa aversão), foi que surgiu o interesse pelo estudo a respeito das atitudes em relação à Matemática correlacionando ao desempenho de alunos do ensino fundamental.

Em relação ao conceito de atitude, há diversas definições de diferentes autores. No entanto, a ideia de atitude adotada na presente pesquisa é conceituada por Brito (1996, p. 11):

"Atitude é definida como uma disposição pessoal, idiossincrática, presente em todos os indivíduos, dirigida a objetos, eventos ou pessoas, que assume diferente direção e intensidade de acordo com as experiências do indivíduo. Além disso, apresenta componentes de domínio afetivo, cognitivo e motor."

Para Viana (2004) atitudes não podem ser diretamente observadas, mas podem ser inferidas pelas respostas avaliativas observadas. Respostas avaliativas são aquelas que expressam aprovação ou desaprovação, como ser favorável ou não, gostar ou não, aproximar ou evitar, atração ou aversão, ou reações similares. Ou seja, as atitudes são inferidas através do comportamento do indivíduo (BRITO, 2006). 
Ainda nesta perspectiva, Aiken e Dreger apud Paula (2008) enfatizam que as atitudes dos professores têm grande influência nas atitudes de seus alunos e em seu desempenho; professores impacientes, hostis e que não dominam o conteúdo, podem influir no surgimento de atitudes negativas no educando. Segundo Paula (2008), no Ensino Fundamental é possível observar que enquanto algumas crianças apresentam dificuldades de aprendizado na Matemática, outras demonstram habilidade e gosto pela disciplina. Para a autora isso não ocorre de maneira inata, mas devido a alguns fatores intervenientes no processo ensino/aprendizagem, como o desenvolvimento de atitude positiva ou negativa em relação à disciplina desde os primeiros anos de escolarização.

As experiências dos alunos em relação à Matemática estão ligadas aos diversos conteúdos aprendidos e a maneira como foram desenvolvidos, à metodologia do professor, aos acontecimentos na sala de aula que causam satisfação ou insatisfação, às formas de avaliação, aos colegas, aos pais, à cultura escolar e à dinâmica da sala de aula, dentre outros fatores que contribuem para o desenvolvimento de atitudes positivas ou negativas que mais tarde irão gerar comportamentos de sucesso ou insucesso na disciplina (VIANA, 2004).

No entanto, as atitudes de uma pessoa não são inatas e nem imutáveis, sendo elas adquiridas e podendo variar com o decorrer do tempo. Daí a grande responsabilidade dos adultos que convivem com a criança e dos educadores na busca de desenvolver atitudes positivas em relação à Matemática e à escola em geral (PAULA, 2008).

$\mathrm{Na}$ teoria dos Paradigmas Bioecológicos de Bronfenbrenner o desenvolvimento do indivíduo é influenciado por uma grande escala de contextos ambientais. Esses contextos são divididos em microssistemas, mesossistema, exossistema, macrossistema e cronossistema, sendo que, a dimensão que a criança em desenvolvimento participa intensamente (e devido a isso a que mais contribui para que ocorra tal desenvolvimento) é o microssistema, onde o sujeito desempenha um padrão de atividades, papéis e relações interpessoais (BROFENBRENNER; MORRIS, apud KREBS, 2001). Assim, caracterizam-se como microssistemas: a escola, a igreja da comunidade, o bairro, o ambiente familiar, o grupo de jovens, a escolinha de esportes, dentre outros ambientes imediatos na vida do indivíduo. Desta maneira, o desenvolvimento de atitudes pode ser influenciado por todas as pessoas que estão inseridas nesses contextos, como os colegas, professores, familiares e outras pessoas que para ele representem referência.

Se as crianças estão inseridas em contextos em que as pessoas possuem atitudes negativas em relação à Matemática, possivelmente, isso irá influenciá-las a também apresentar atitudes similares, contribuindo para que estas não tenham um aprendizado efetivo. Para Eagly e Chaiken citado por Viana (2004), uma atitude é uma ação psicológica expressa quando o indivíduo avalia algo demonstrando ser favorável ou desfavorável a ela. 
Com base nesses pressupostos tornam-se importantes, iniciativas de estudos que tentem investigar os fatores intervenientes na aprendizagem do aluno, não só na Matemática, mas em todo o currículo escolar. No entanto, além da falta de pesquisas relativas ao tema na região, o presente estudo se justifica devido a repulsa de muitos escolares em relação à Matemática observada nas escolas, o que pode contribuir para o grande número de reprovações na disciplina, como também, o não entendimento dos conteúdos mesmo naqueles os quais conseguem aprovação.

Assim, o objetivo deste trabalho foi verificar se as atitudes em relação à Matemática poderiam estar relacionadas ao desempenho de estudantes do 60 ano do Ensino Fundamental na referida disciplina.

\section{Material e métodos}

O presente estudo se caracteriza como uma pesquisa descritiva correlacional, transversal, quantitativa, observacional e de campo. A população estudada foi de estudantes do 60 ano do Ensino Fundamental da rede pública de ensino do município de Caririaçu, interior do Ceará. A amostra foi do tipo intencional e não probabilística composta por 37 sujeitos de ambos os sexos com idades entre 10 e 14 anos, matriculados na Escola de Ensino Fundamental e Médio São Pedro. Foi entregue a todos os participantes da pesquisa o Termo de Consentimento Livre e Esclarecido (TCLE) a fim de registrar a autorização dos pais para a participação das crianças na mesma.

Para coleta de dados utilizou-se a Escala de Atitudes em Relação à Matemática (EARM) elaborada por Aiken e adaptada por Brito (1996) a fim de investigar sentimentos negativos (afirmações 01, 02, 06, 07, 08, 09, 10, 12, 13 e 17) ou positivos (afirmações 03, 04, 05, 09, 11, 15, $16,19,20)$ em relação à Matemática (ANEXO). Este instrumento é do tipo escala de Likert composto por vinte questões, sendo dez afirmações positivas e dez negativas, além de uma questão para o sujeito avaliar o seu desempenho em relação à Matemática. Para cada afirmação, atribui-se uma pontuação de um a quatro, e o escore total pode variar de 20 a 80 pontos (PAULA, 2008). Para afirmações positivas, a ordem de atribuição dos valores foi 1, 2, 3 e 4. Para afirmações negativas, a pontuação tinha ordem inversa, ou seja, 4, 3, 2 e 1 . O escore do questionário foi obtido a partir da soma dos pontos das vinte questões. Para avaliar o desempenho escolar na disciplina de Matemática utilizou-se a média das notas dos três primeiros bimestres, pois a pesquisa foi desenvolvida antes do término do ano letivo.

A análise estatística se deu com a confecção de um banco de dados em pacote estatístico. Utilizou-se estatística descritiva de média, desvio padrão e distribuição de frequência. A análise inferencial se deu com o teste $t$ de Student para comparar o resultado do Questionário de Atitudes em Relação à Matemática entre meninos e meninas. Além disso, foi utilizado o teste de 
correlação de Spearman para avaliar o grau de associação entre as atitudes em relação à Matemática e o desempenho escolar na disciplina. Para ambos os testes o nível de confiança foi de $5 \%$.

\section{Análise e discussão dos resultados}

A apresentação dos resultados se dará primeiramente com alguns dados descritivos da amostra no que diz respeito às idades e ao gênero, e posteriormente a apresentação do resultado dos questionários e do desempenho dos alunos na disciplina de Matemática. A tabela 1 apresenta a distribuição de frequência dos sujeitos submetidos ao estudo.

Tabela 1 - Distribuição de frequência da amostra em função do gênero e idade

\begin{tabular}{|c|c|c|c|c|c|}
\hline & $\mathbf{1 0}$ anos & $\mathbf{1 1}$ anos & $\mathbf{1 2}$ anos & $\mathbf{1 3}$ anos & $\mathbf{1 4}$ anos \\
\hline Feminino & 2 & 7 & 3 & 2 & 4 \\
& $11,1 \%$ & $38,9 \%$ & $16,7 \%$ & $11,1 \%$ & $22,2 \%$ \\
\hline \multirow{2}{*}{ Masculino } & 1 & 4 & 6 & 4 & 4 \\
& $5,3 \%$ & $21,1 \%$ & $31,6 \%$ & $21,1 \%$ & $21,1 \%$ \\
\hline \multirow{2}{*}{ TOTAL } & 3 & 11 & 9 & 6 & 8 \\
& $8,1 \%$ & $29,7 \%$ & $24,3 \%$ & $16,2 \%$ & $21,6 \%$ \\
\hline
\end{tabular}

Nota-se que quanto ao gênero, 18 (48,6\%) eram meninas e 19 (51,4\%) eram meninos, sendo a maioria (54\%) apresentaram-se com 11 e 12 anos de idade. Os sujeitos apresentaram uma média de idade de $12,1 \pm 1,2 \mathrm{dp}$ anos, sendo $11,9 \pm 1,3 \mathrm{dp}$ anos para as meninas e $12,3 \pm 1,2$ dp para os meninos, não sendo apontadas diferenças significativas entre os gêneros no que diz respeito à idade $(p=0,391)$.

A seguir, a tabela 2 apresenta as médias da pontuação do questionário aplicado para avaliar as atitudes em relação à Matemática e das notas obtidas durante os três primeiros bimestres do ano pelos alunos na disciplina. No que diz respeito ao EARM as meninas apresentaram uma média um pouco superior ao resultado obtido pelos meninos, no entanto, tais diferenças não foram apontadas como estatisticamente significativas pelo teste $t$ de Student, como pode ser observado na tabela. No que se trata do desempenho dos alunos na disciplina, novamente as meninas apresentaram resultados mais elevados, sendo que nesta variável, o teste $t$ apontou diferenças significativas a favor deste grupo. 
Tabela 2 - Média e desvio padrão do resultado da EARM e das notas dos

alunos na disciplina de Matemática

\begin{tabular}{|c|c|c|c|c|}
\hline & EARM & $\begin{array}{c}\text { teste } t \text { de } \\
\text { Student }\end{array}$ & Média das notas & $\begin{array}{c}\text { teste } t \text { de } \\
\text { Student }\end{array}$ \\
\hline Feminino & $\frac{64,2 \pm 11,9 \mathrm{dp}}{62,7 \pm 8,2 \mathrm{dp}} 0,673$ & $\frac{7,5 \pm 1,1 \mathrm{dp}}{6,4 \pm 1,1 \mathrm{dp}} * 0,007$ \\
Masculino & $63,4 \pm 10,1 \mathrm{dp}$ & $6,9 \pm 1,2 \mathrm{dp}$ \\
\hline TOTAL & \multicolumn{2}{|c|}{6} & \multicolumn{2}{c|}{6} \\
\hline
\end{tabular}

Em relação à média geral da EARM, os resultados aqui encontrados se aproximam ao do estudo realizado por Paula (2008) com 22 estudantes do quinto ano do Ensino Fundamental do município de São José do Rio Preto. No referido estudo os sujeitos apresentaram uma média de 64,05 e desvio padrão de 9,91 contra $63,48 \pm 10,11$ da presente pesquisa. O resultado obtido pelos estudantes da cidade de Caririaçu torna-se mais significativo ainda quando comparado ao estudo de Jesus (2005) com alunos também do 60 ano do Ensino Fundamental, onde apresentaram uma média de 58,5 na escala de atitudes em relação à Matemática. A média obtida pelos sujeitos neste trabalho também são superiores aos achados de Brito (1996), Gonçalez (2000), de Viana (2005) e Araújo (1999). No entanto, tais estudos foram realizados com populações diferentes em comparação ao presente estudo. Ainda assim, se supõem que os achados desta pesquisa evidenciam que os alunos apresentam atitudes favoráveis no que diz respeito à Matemática.

Tabela 3 - Distribuição de frequência das respostas dadas em cada uma das afirmações da escala de atitudes em relação à Matemática

\begin{tabular}{|c|c|c|c|c|}
\hline AFIRMAÇÃo & $\begin{array}{c}\text { Discordo } \\
\text { Totalmente }\end{array}$ & Discordo & Concordo & $\begin{array}{c}\text { Concordo } \\
\text { Totalmente }\end{array}$ \\
\hline $\begin{array}{c}\text { 01- Eu fico sempre sob uma } \\
\text { terrível tensão na aula de } \\
\text { Matemática. }\end{array}$ & $\mathbf{3 7 , 8 \%}$ & $27 \%$ & $18,8 \%$ & $24,3 \%$ \\
\hline $\begin{array}{c}\text { 02- Eu não gosto de Matemática } \\
\text { e me assusta ter que fazer essa } \\
\text { matéria. }\end{array}$ & $\mathbf{6 7 , 6 \%}$ & $21,6 \%$ & $8,1 \%$ & $2,7 \%$ \\
\hline $\begin{array}{c}\text { 03- Eu acho a Matemática muito } \\
\text { interessante e gosto das aulas de } \\
\text { Matemática. }\end{array}$ & $5,4 \%$ & $24,3 \%$ & $\mathbf{7 0 , 3 \%}$ \\
\hline
\end{tabular}




\begin{tabular}{|c|c|c|c|c|}
\hline $\begin{array}{l}\text { 04- A Matemática é fascinante e } \\
\text { divertida. }\end{array}$ & $13,5 \%$ & $8,1 \%$ & $10,8 \%$ & $67,6 \%$ \\
\hline $\begin{array}{l}\text { 05- A Matemática me faz sentir } \\
\text { seguro (a) e é, ao mesmo tempo, } \\
\text { estimulante. }\end{array}$ & $13,5 \%$ & & $43,2 \%$ & $43,2 \%$ \\
\hline $\begin{array}{l}\text { 06- "Dá um branco" na minha } \\
\text { cabeça e não consigo pensar } \\
\text { claramente quando estudo } \\
\text { Matemática. }\end{array}$ & $51,4 \%$ & $16,2 \%$ & $27 \%$ & $5,4 \%$ \\
\hline $\begin{array}{l}\text { 07- Eu tenho sensação de } \\
\text { insegurança quando me esforço } \\
\text { em Matemática. }\end{array}$ & $37,8 \%$ & $40,5 \%$ & $13,5 \%$ & $8,1 \%$ \\
\hline $\begin{array}{l}\text { 08- A Matemática me deixa } \\
\text { inquieto (a), descontente, } \\
\text { irritado (a) e impaciente. }\end{array}$ & $51,4 \%$ & $18,9 \%$ & $5,4 \%$ & $24,3 \%$ \\
\hline $\begin{array}{l}\text { 09- O sentimento que tenho com } \\
\text { relação à Matemática é bom. }\end{array}$ & $5,4 \%$ & $2,7 \%$ & $32,4 \%$ & $59,5 \%$ \\
\hline $\begin{array}{l}\text { 10- A Matemática me faz sentir } \\
\text { como se estivesse perdido (a) em } \\
\text { uma selva de números e sem } \\
\text { encontrar a saída. }\end{array}$ & $48,6 \%$ & $27 \%$ & $16,2 \%$ & $8,1 \%$ \\
\hline $\begin{array}{l}\text { 11- A Matemática é algo que eu } \\
\text { aprecio grandemente. }\end{array}$ & $16,2 \%$ & $10,8 \%$ & $27 \%$ & $45,9 \%$ \\
\hline $\begin{array}{l}\text { 12- Quando eu ouço a palavra } \\
\text { Matemática, eu tenho um } \\
\text { sentimento de aversão. }\end{array}$ & $59,5 \%$ & $18,9 \%$ & $5,4 \%$ & $16,2 \%$ \\
\hline $\begin{array}{l}\text { 13- Eu encaro a Matemática com } \\
\text { um sentimento de indecisão, que } \\
\text { é resultado do medo de não ser } \\
\text { capaz em Matemática. }\end{array}$ & $35,1 \%$ & $24,3 \%$ & $27 \%$ & $13,5 \%$ \\
\hline $\begin{array}{l}\text { 14- Eu gosto realmente da } \\
\text { Matemática. }\end{array}$ & $8,1 \%$ & $2,7 \%$ & $27 \%$ & $62,2 \%$ \\
\hline 15- A Matemática é uma das & $10,8 \%$ & $10,8 \%$ & $24,3 \%$ & $54,1 \%$ \\
\hline
\end{tabular}

R. B. E. C. T., vol 6, núm. 3, set-dez.2013 ISSN - 1982-873X 


\begin{tabular}{|c|c|c|c|c|}
\hline $\begin{array}{c}\text { matérias que eu realmente gosto } \\
\text { de estudar na escola. }\end{array}$ & 27\% & $21,6 \%$ & $\mathbf{3 2 , 4 \%}$ & $\mathbf{1 8 , 9 \%}$ \\
\hline $\begin{array}{c}\text { 16- Pensar sobre a obrigação de } \\
\text { resolver um problema } \\
\text { matemático me deixa nervoso. }\end{array}$ & $\mathbf{7 0 , 3 \%}$ & $16,2 \%$ & $5,4 \%$ & $\mathbf{8 , 1 \%}$ \\
\hline $\begin{array}{c}\text { Matemática e é a matéria que } \\
\text { me dá mais medo. }\end{array}$ & $24,3 \%$ & $18,9 \%$ & $24,3 \%$ & $\mathbf{3 2 , 4 \%}$ \\
\hline $\begin{array}{c}\text { 18- Eu fico mais feliz na aula de } \\
\text { Matemática que na aula de } \\
\text { qualquer outra matéria. }\end{array}$ & $8,1 \%$ & $5,4 \%$ & $24,3 \%$ & $\mathbf{6 2 , 2} \%$ \\
\hline $\begin{array}{c}\text { 19- Eu me sinto tranquilo (a) em } \\
\text { Matemática e gosto muito dessa } \\
\text { matéria. }\end{array}$ & $10,8 \%$ & $2,7 \%$ & $25,1 \%$ & $\mathbf{5 1 , 4 \%}$ \\
\hline $\begin{array}{c}\text { 20- Eu tenho uma reação } \\
\text { definitivamente positiva com } \\
\text { relação à Matemática: Eu gosto e } \\
\text { aprecio essa matéria. }\end{array}$ & & & & \\
\hline
\end{tabular}

É significante notar que $94,6 \%$ dos participantes consideram a Matemática interessante e que gostam da disciplina, como mostra a afirmação 3 . Além disso, $86,5 \%$ da amostra acham que a disciplina é fascinante e divertida, e se sentem tranquilos em relação à Matemática. No geral, o que se pode observar diante das respostas dos alunos, é que a grande maioria deles possuem atitudes positivas em relação à Matemática, inclusive na afirmação 18, na qual a maioria $(56,7 \%)$ dos sujeitos afirmam se sentirem mais felizes na aula de Matemática do que em qualquer outra disciplina. Evidencia-se que para a maior parte desses alunos, as atitudes em relação à disciplina são positivas.

Estes achados corroboram com a pesquisa de Paula (2008), na qual 100\% da amostra consideraram a Matemática interessante, fascinante e divertida, e quase a totalidade da amostra $(95,5 \%)$ se sentem tranquilos e gostam da matéria. No estudo de validação do EARM, realizado com estudantes do Ensino Fundamental e Médio, os resultados foram mais contraditórios com a maioria da amostra apresentando atitudes positivas em algumas afirmações e negativas em outras (BRITO, 1996). Estas discrepâncias podem ter ocorrido devido às diferenças das faixas etárias dos participantes dos estudos. Talvez, os sujeitos de maior idade tenham uma melhor percepção de competência em relação aos mais novos, o que pode influenciar na crença 
de autoeficácia dos mesmos. Possivelmente, alunos que se percebem com pouca competência podem ter uma baixa crença de autoeficácia, ficando assim ansiosos em relação à disciplina e gerando atitudes negativas com a mesma.

No gráfico 1 pode-se observar que quanto maior a pontuação na EARM, maior é o desempenho dos alunos nas médias das notas durante o decorrer do ano, sendo que apesar de o teste de correlação de Spearman apontar uma correlação positiva, esta apresentou-se apenas de forma moderada (ró=0,579). No entanto, o mais interessante encontrado é que quando tal associação foi analisada separadamente entre os gêneros masculino e feminino, observou-se que no sexo masculino a correlação foi fraca (ró=0,343), enquanto no feminino foi apontada uma correlação considerada forte $(r o ́=0,758)$.

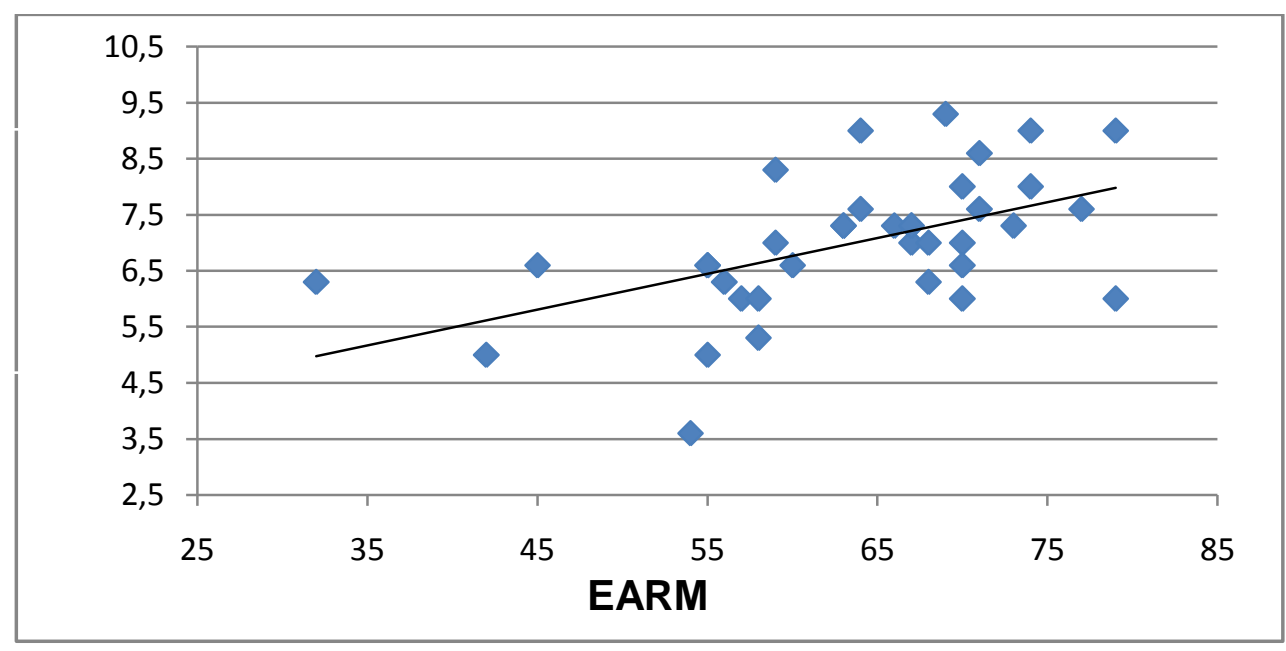

Gráfico 1 - Gráfico de dispersão entre as atitudes em relação à

Matemática e o desempenho dos alunos

Os achados deste estudo no que diz respeito a esta associação corroboram com alguns estudos já realizados no Brasil onde também evidenciam associações entre as atitudes e o desempenho. Por exemplo: no estudo de Jesus (2005), onde correlacionava as atitudes com o desempenho na resolução de algumas operações aritméticas; e com a pesquisa de Gonçalez (2000), a qual mostrou que a maioria dos sujeitos que obtinham boas notas durante o ano apresentavam atitudes positivas em relação à Matemática (PAULA, 2008). Ainda mais, numa pesquisa realizada envolvendo as mesmas variáveis com educação de jovens e adultos, também se observou relação positiva e moderada entre as atitudes e o desempenho (REFOSCO, et al., 2004). 


\section{Considerações finais}

Os achados do presente estudo vão de encontro à literatura vigente sobre o assunto no país, evidenciando uma relação positiva entre as atitudes em relação à Matemática e o desempenho escolar na disciplina, apesar de a correlação se apresentar de forma moderada. No entanto, o fato curioso diz respeito à maior correlação do grupo feminino na relação das variáveis estudadas, que pode ser explicada pelas melhores notas na disciplina do grupo feminino, como foi verificado pelo teste $t$ de Student que apontou diferenças significativas entre os gêneros.

No entanto, não existem referências que afirmem que o gênero seja um fator determinante no desempenho cognitivo e matemático. Todavia, existem diversos fatores além das atitudes em relação à disciplina que contribuem diretamente no processo de aprendizagem do aluno, tanto de ordem genética como aspectos ambientais os quais não foram aqui estudados.

Sugere-se que para futuros estudos sejam utilizadas amostras maiores e de séries diferentes para que se possa investigar possíveis mudanças de atitudes entre as faixas etárias, assim como alguns fatores ambientais que podem influenciar o desempenho escolar do aluno e que geralmente são negligenciados pelo sistema educacional.

\section{Referências Bibliográficas}

ARAUJO, E. A. (1999). Influências das habilidades e das atitudes em relação à Matemática e a escolha profissional.Tese de Doutorado, Faculdade de Educação da Universidade Estadual de Campinas - UNICAMP, Campinas, SP.

BRITO, M. R. F. Um estudo sobre as atitudes em relação à Matemática em estudantes de 10 e 20 graus. Tese de Livre Docência, UNICAMP/FE, Campinas, SP, 1996.

BRITO, M. R. F. Atitudes, ansiedade, afeto e Matemática. Anais do XIX Encontro Nacional de Professores do PROEPRE. Águas de Lindóia. 81 - 93, 2002.

BRITO, M.R.F. Solução de Problemas e a Matemática escolar. Campinas: Alínea, v.1, p.185-205, 2006.

FIORENTINI, Dário; MIORIM, Maria Ângela. Uma reflexão sobre o uso de materiais concretos e jogos no ensino da Matemática. Boletim da SBEM, v.2, n. 7, p. 7-36, jul-ago, 1990.

KREBS, R. J. Novas Tendências para o estudo do desenvolvimento humano. Coleção Prata da Casa (UFMA), São Luís-MA, n. 11, p. 93-108, 2001.

GONÇALEZ, M. H. C. Relações entre família, gênero, desempenho, confiança e atitudes em relação à Matemática. Tese de Doutorado,Faculdade de Educação da Universidade Estadual de Campinas - UNICAMP, Campinas, SP, 2000. 
LOOS, H; FALCÃO, J. T. R. e REGNIER, N. M. A. (2001). A ansiedade na aprendizagem da Matemática e a passagem da aritmética para álgebra. InMárcia R. F. Brito (Org.) Psicologia da educação Matemática: teoria epesquisa. Florianópolis: Ed. Insular, 235-261.

JESUS, M. A. S. As Atitudes e o Desempenho em Operações Aritméticas do Ponto de Vista da Aprendizagem Significativa. Tese de Doutorado, Faculdade de Educação da Universidade Estadual de Campinas - UNICAMP, Campinas, SP, 2005.

PAULA, K. C. M. A família, o desenvolvimento das atitudes em relação à Matemática e a crença de auto-eficácia. Dissertação de Mestrado. Faculdade de Educação da Universidade Estadual de Campinas - UNICAMP. Campinas, 2008.

REFOSCO, M. I.; MENDES, C. R.; ROGOVSKI, I. As atitudes em relação à Matemática e o desempenho matemático e algébrico na educação de jovens e adultos. In: 27ạ Reunião anual da ANPED, 2004, Caxambu. 27ạ Reunião anual da ANPED, 2004.

SANCHEZ, J. N. G. Dificuldades de Aprendizagem e Intervenção Psicopedagógica. Porto Alegre: Artmed, 2004.

VIANA, O. A. As atitudes de alunos do ensino médio em relação à geometria: adaptação e validação de escala. In: XVIII Encontro de Educação Matemática. Universidade Federal do Pernambuco, 2004.

VIANA, O. A. O Componente Espacial da Habilidade Matemática de Alunos do Ensino Médio e as Atitudes em Relação à Matemática e à Geometria. Tese de Doutorado, Faculdade de Educação da Universidade Estadual de Campinas - UNICAMP, Campinas, SP, 2005.

Cicero Luciano Alves Costa: Professor do Curso de Licenciatura em Educação Física da Faculdade de Ciências Aplicadas Doutor Leão Sampaio. luciano.alvescosta@yahoo.com.br

Cicera Vania Barros Costa: Professora da rede Estadual de Ensino do Ceará. vania mat5@hotmail.com 